\title{
EFEITO DE ANTIOXIDANTES SOBRE O ESCURECIMENTO DE BATATAS BAROA MINIMAMENTE PROCESSADAS
}

Lara Santana Fernandes ${ }^{1}$, Paulo Cesar Corrêa ${ }^{2}$, Fernando Luiz Finger ${ }^{3}$, Mateus da Silva Junqueira ${ }^{4}$, Kelem Silva Fonseca $^{5}$

\section{RESUMO}

Objetivou-se com este trabalho avaliar o efeito de diferentes concentrações dos antioxidantes ácido ascórbico e ácido cítrico sobre o escurecimento de batatas baroa minimamente processadas. As raízes foram selecionadas, lavadas com detergente e enxaguadas em água corrente. Em seguida, foram sanitizadas, descascadas, cortadas em fatias de $1 \mathrm{~cm}$ de espessura e novamente sanitizadas com posterior enxágue. Logo após, as rodelas foram imersas nos seguintes tratamentos: água gelada; solução de ácido ascórbico $1 \%$ + ácido cítrico $1 \%$; solução de ácido ascórbico 2\% + ácido cítrico $2 \%$ e solução de ácido ascórbico $3 \%+$ ácido cítrico $3 \%$. As rodelas foram centrifugadas, acondicionadas em embalagens de polipropileno e mantidas a $5 \pm 2{ }^{\circ} \mathrm{C}$ e $90 \pm 5 \%$ de umidade relativa, durante um período de oito dias. Para a avaliação da perda de massa, o experimento foi instalado em esquema fatorial 4 x 9 (4 tratamentos aos 0 , 1 , 2, 3, 4, 5, 6, 7 e 8 dias) no delineamento inteiramente casualizado com quatro repetições. Para as análises de cor, $\mathrm{pH}$ e teor de sólidos solúveis foi utilizado um esquema fatorial 4 × 5 (4 tratamentos aos 0, 2, 4, 6 e 8 dias) com quatro repetições. Os dados foram submetidos à metodologia de superfície de resposta. As batatas apresentaram melhor qualidade com o tratamento 3\% ácido ascórbico + 3\% ácido cítrico, chegando ao final do armazenamento com menor perda da coloração amarela.

Palavras-chave: Concentração ácida, armazenamento, processamento mínimo, Arracacia xanthorrhiza

\section{ABSTRACT}

\section{EFFECT OF ANTIOXIDANTS ON BROWNING OF FRESH-CUT YELLOW PERUVIAN ROOTS}

The objective of this study was to evaluate the effect of different concentrations of the antioxidants ascorbic acid and citric acid on browning of fresh-cut yellow peruvian roots. After selection, the roots were washed with detergent and rinsed in running water. They were then sanitized, peeled, cut into $1 \mathrm{~cm}$ thick slices and sanitized again, with subsequent rinsing. After that, the slices were immersed in the following treatments: cold water; $1 \%$ ascorbic acid +1 $\%$ citric acid solution; $2 \%$ ascorbic acid $+2 \%$ citric acid solution; and $3 \%$ ascorbic acid $+3 \%$ citric acid solution. The slices were centrifuged, packed in polypropylene packaging and stored at $5 \pm 2{ }^{\circ} \mathrm{C}$ and $90 \pm 5 \%$ relative humidity, for a period of eight days. For evaluation of mass loss, the experiment was setup as a 4 x 9 factorial ( 4 treatments at $0,1,2$, $3,4,5,6,7$, and 8 days of storage) in a completely randomized design with four repetitions. For analysis of color, $\mathrm{pH}$ and soluble solids, a 4 x 5 factorial design was constructed (4 treatments at 0, 2, 4, 6 and 8 days of storage), with four repetitions. The collected data was submitted to the surface response methodology. Roots presented a better quality when treated with 3\% ascorbic acid $+3 \%$ citric acid solution, reaching the end of storage with lower yellow color loss.

Keywords: Acid concentration, storage, fresh cut, Arracacia xanthorrhiza

\section{Recebido para publicação em 29/04/2013. Aprovado em 12/12/2013.}

1 - Eng. de Alimentos, Doutoranda em Engenharia Agrícola, UFV/Viçosa-MG, lara.santanaf@gmail.com

2 - Professor Associado, Departamento de Engenharia Agrícola, UFV/Viçosa-MG, copace@ufv.br

3 - Professor Associado, Departamento de Fitotecnia, UFV/Viçosa-MG, ffinger@ufv.br

4 - Professor Adjunto, Departamento de Engenharia de Alimentos, UFES-ES, mateusjunq@yahoo.com.br

5 - Eng. Agrônoma, Doutoranda em Fisiologia Vegetal, UFV/Viçosa-MG, kelemsilva@yahoo.com.br 


\section{INTRODUÇÃO}

Amudança nos padrões de consumo de alimentos tem levado à maior demanda por frutas e hortaliças em detrimento dos produtos industrializados. Ao mesmo tempo, os consumidores desejam produtos com qualidade e praticidade. Nesse sentido, a procura por frutas e hortaliças minimamente processadas tem evoluído rapidamente (ARRUDA et al., 2004).

Produtos minimamente processados são aqueles submetidos a uma ou mais etapas de préprocessamento, como lavagem, descascamento, fatiamento e corte; e, em alguns casos, a tratamentos químicos, tornando-os prontos para o consumo ou preparo, mas mantidos em estado fresco e metabolicamente ativos, com propósito de modificar a sua apresentação para consumo (MORETTI, 2007).

Uma das desvantagens desta hortaliça é a sua baixa capacidade de conservação pós-colheita, com um período máximo de armazenamento de seis dias em temperatura ambiente $\left(25^{\circ} \mathrm{C}\right)$ e $40 \%$ de umidade relativa (SCALON et al.,2002). Esta alta perecibilidade das raízes gera dificuldades no armazenamento, o que tem despertado o interesse para a diversificação de seu uso na forma processada. Para tornar esse tipo de alimento menos perecível, destaca-se o uso de antioxidantes, durante a etapa de processamento, associado à refrigeração (LUPETTI et al., 2005).

As reações de escurecimento são alguns dos fenômenos mais importantes que ocorrem durante o processamento mínimo da batata baroa (NUNES et al., 2011). O escurecimento, além de depreciar a qualidade do produto, leva também ao desenvolvimento de sabores desagradáveis e perdas na qualidade nutricional (HAMINIUK et al., 2005). O uso de antioxidantes ou agentes anti-escurecimento, como também são chamados, torna-se uma alternativa para controlar o problema do escurecimento (PINELI et al., 2005).

Dentre os antioxidantes mais pesquisados destacam-se os ácidos cítrico e ascórbico, por sua disponibilidade no mercado e também por seu amplo uso na indústria alimentícia (PINELI et al., 2005).

Em função da depreciação causada pelo escurecimento das batatas baroa minimamente processadas, objetivou-se com este trabalho avaliar o efeito de diferentes concentrações dos antioxidantes ácido ascórbico e ácido cítrico, visando prolongar a vida útil do produto final.

\section{MATERIAL E MÉTODOS}

O presente trabalho foi desenvolvido no Laboratório de Propriedades Físicas e Qualidade de Produtos Agrícolas pertencente ao Centro Nacional de Treinamento em Armazenagem (CENTREINAR), e na Unidade de Processamento Mínimo, da Universidade Federal de Viçosa (UFV), MG.

Para realização dos experimentos foram utilizadas batatas baroa da cultivar Amarela de Carandaí. Foram eliminados os tubérculos danificados com a finalidade de se obter um produto homogêneo.

No processamento mínimo, as batatas foram selecionadas e lavadas em água corrente com o auxílio de uma escova para a eliminação de sujidades. Em seguida, elas foram imersas em uma solução com $200 \mathrm{mg} \mathrm{L}^{-1}$ de cloro ativo, a 5 $\pm 2{ }^{\circ} \mathrm{C}$, por $15 \mathrm{~min}$. $\mathrm{O}$ sanitizante utilizado tem como princípio ativo o dicloro s-triazinatriona sódica dihidratada $\left(\right.$ Sumaveg $\left.^{\circledR}\right)$. Em seguida, as batatas foram descascadas, cortadas manualmente em rodelas na espessura de $1 \mathrm{~cm}$ e colocadas imediatamente em água gelada.

Para a sanitização final, as rodelas de batatas baroa foram imersas em solução contendo $200 \mathrm{mg}$ $\mathrm{L}^{-1}$ de cloro ativo, a $5 \pm 2{ }^{\circ} \mathrm{C}$, por $15 \mathrm{~min}$, com posterior enxágue em solução contendo $3 \mathrm{mg} \mathrm{L}^{-1} \mathrm{de}$ cloro ativo, a $5 \pm 2{ }^{\circ} \mathrm{C}$ por $15 \mathrm{~min}$. O processamento prosseguiu com imersão das rodelas, por $5 \mathrm{~min}$, nos seguintes tratamentos químicos: TE: testemunha (imersão em água gelada); TR 1: solução de ácido ascórbico (AA) $1 \%$ + ácido cítrico (AC) 1\%; TR 2: solução de ácido ascórbico (AA) $2 \%+$ ácido cítrico (AC) 2\% e TR 3: solução de ácido ascórbico (AA) 3\% + ácido cítrico (AC) 3\%.

$\mathrm{O}$ excesso de água das rodelas de batatas baroa foi retirado por centrifugação a $2000 \mathrm{rpm}$ por $15 \mathrm{~s} \mathrm{e}$ as rodelas foram acondicionadas em embalagens de polipropileno de $10 \mu \mathrm{m}$ de espessura e dimensões de 16 x $25 \mathrm{~cm}$, seladas a quente com auxílio de uma seladora. Durante o armazenamento, as 
amostras foram mantidas a $5 \pm 2{ }^{\circ} \mathrm{C}$ e $90 \pm 5 \%$ de umidade relativa (UR), por um período de oito dias, procedendo-se as análises físico-químicas, a cada dois dias.

A caracterização da cor das rodelas de batatas baroa minimamente processada foi feita com o auxílio de um colorímetro tristímulo, com leitura direta de reflectância das coordenadas $\mathrm{L}^{*}$ (luminosidade), a* (tonalidades vermelha/verde) e $\mathrm{b}^{*}$ (tonalidades amarela/azul), empregando a escala Hunter-Lab e utilizando o iluminante $10^{\circ} / \mathrm{D} 60$.

A partir dos valores de $L^{*}, a^{*}$ e $b^{*}$, foram calculados os seguintes índices colorimétricos: o croma $\left(\mathrm{C}^{*}\right)$, que define a intensidade e a pureza de uma cor (Equação 1); e o ângulo hue $\left(\mathrm{h}^{*}\right)$, que define a tonalidade da cor (Equação 2): $0^{\circ}$ (vermelho), $90^{\circ}$ (amarelo), $180^{\circ}$ (verde) e $270^{\circ}$ (azul). O $\Delta$ E, que é a diferença total de cor levando-se em consideração as condições iniciais de armazenamento do produto, foi calculado pela Equação 3.

$$
\begin{aligned}
& C^{*}=\left(a^{* 2}+b^{* 2}\right)^{1 / 2} \\
& h^{*}=\tan ^{-1} \frac{b^{*}}{a^{*}}
\end{aligned}
$$

$$
\Delta E=\left(\Delta L^{* 2}+\Delta a^{* 2}+\Delta b^{* 2}\right)^{1 / 2}
$$

Outros parâmetros estudados foram o índice de escurecimento (IE) e o índice de amarelecimento (YI), calculados respectivamente pelo modelo proposto por Palou et al. (1999) e metodologia descrita na ASTM D1925, citada por Andrady e Torikai (1999), Equações 4, 5 e 6:

$$
\begin{aligned}
& I E=\frac{100(X-0,31)}{0,172} \\
& X=\frac{a^{*}+1,75 L^{*}}{5,64 L^{*}+a^{*}-3,01 b^{*}} \\
& Y I=\frac{100\left(0.72 a^{*}+1,79 b^{*}\right)}{L^{*}}
\end{aligned}
$$

$\mathrm{O}$ pH foi determinado diretamente nas amostras por potenciometria, utilizando-se um pHmetro digital,conforme AOAC (1997).

O teor de sólidos solúveis foi determinado pela leitura direta com o auxílio de um refratômetro, e os resultados foram expressos em ${ }^{\circ}$ Brix, conforme AOAC (1997).

A perda de massa foi determinada por meio da diferença de massa no tempo analisado em relação ao tempo zero (Equação 7). As pesagens foram realizadas diariamente.

$$
P=\frac{M_{(t=0)}-M_{(t=t)}}{M_{(t=0)}} \times 100
$$

em que,

$\mathrm{P}=$ perda de água, $\%$;

$\mathrm{M}_{(\mathrm{t}=0)}=$ massa da batata baroa no tempo zero, $\mathrm{g}$;

$\mathrm{M}_{(\mathrm{t} t \mathrm{t})}=$ massa da batata baroa no tempo $\mathrm{t}, \mathrm{g}$.

Para a avaliação da perda de massa o experimento foi instalado, em esquema fatorial $4 \mathrm{x}$ 9 , sendo 4 tratamentos químicos (TE- testemunha, TR 1- solução de ácido ascórbico 1\% + ácido cítrico $1 \%$, TR 2- solução de ácido ascórbico $2 \%$ + ácido cítrico $2 \%$ e TR 3- solução de ácido ascórbico 3\% + ácido cítrico 3\%) e 9 períodos de armazenamento $(0,1,2,3,4,5,6,7$ e 8 dias) no delineamento inteiramente casualizado com quatro repetições.

Para as demais análises foi feito um esquema fatorial 4 × 5 (4 tratamentos aos $0,2,4,6$ e 8 dias) com quatro repetições. Os dados foram submetidos à análise utilizando-se o método da superfície de resposta. Os modelos foram escolhidos baseados na significância dos coeficientes de regressão utilizando-se o teste " $t$ ", adotando o nível de $5 \%$ de probabilidade no coeficiente de determinação $\left(\mathrm{R}^{2}\right)$ e no comportamento fisiológico.

\section{RESULTADOS E DISCUSSÃO}

No Quadro 1 estão os resultados médios das coordenadas $\mathrm{L}^{*}, \mathrm{a}^{*} \mathrm{e} \mathrm{b}^{*}$ referente ao espectro de cor. 
Quadro 1. Valores das coordenadas $L^{*}$, $a^{*}$ e $b^{*}$ das rodelas de batatas baroa imersas em água (TE) e nas soluções: AA 1\% + AC 1\% (TR1), AA 2\% + AC 2\% (TR2) e AA 3\% + AC 3\% (TR3), ao longo do armazenamento

\begin{tabular}{|c|c|c|c|c|c|c|c|c|c|c|c|c|}
\hline \multirow{2}{*}{$\begin{array}{c}\text { Tempo } \\
\text { (dias) }\end{array}$} & \multicolumn{4}{|c|}{$\mathrm{L}^{*}$} & \multicolumn{4}{|c|}{$a^{*}$} & \multicolumn{4}{|c|}{$b^{*}$} \\
\hline & TE & TR1 & TR2 & TR3 & $\mathrm{TE}$ & TR1 & TR2 & TR3 & TE & TR1 & TR2 & TR3 \\
\hline 0 & 59,95 & & & & 7,53 & & & & 40,98 & & & \\
\hline 2 & 63,87 & 63,62 & 64,51 & 64,5 & 7,26 & 7,53 & 7,65 & 7,82 & 36,99 & 38,89 & 41,79 & 41,76 \\
\hline 4 & 63,85 & 64,65 & 63,99 & 64,64 & 7,23 & 7,43 & 7,57 & 7,70 & 33,14 & 32,64 & 37,35 & 39,37 \\
\hline 6 & 64,19 & 64,11 & 65,63 & 66,22 & 7,31 & 7,63 & 7,73 & 8,00 & 42,40 & 29,18 & 35,85 & 35,65 \\
\hline 8 & 66,05 & 64,01 & 64,95 & 64,96 & 7,79 & 8,03 & 8,13 & 8,35 & 31,93 & 28,91 & 31,70 & 34,67 \\
\hline
\end{tabular}

Na Figura 1 é mostrada a evolução dos valores de L* ao longo do período de armazenamento do produto.

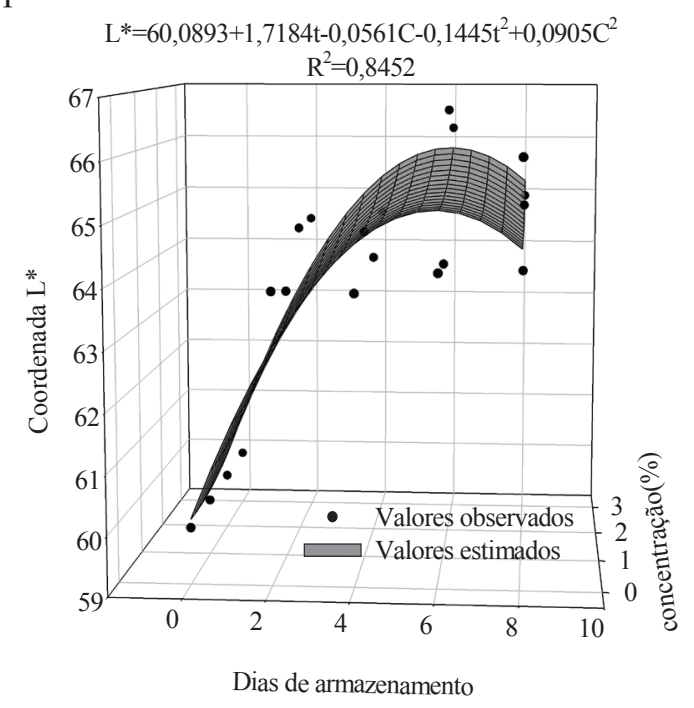

(a)

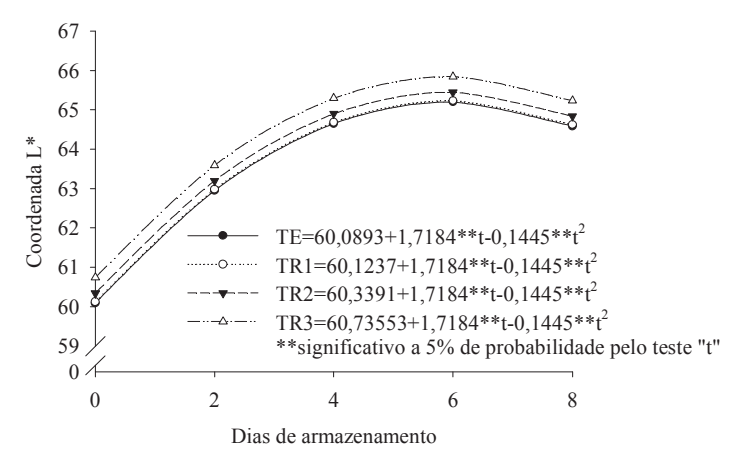

(b)

Figura 1. (a) Superfície de resposta da coordenada L*. (b) Cortes da superfície de resposta da coordenada $\mathrm{L}^{*}$ para os tratamentos (TE, TR1, TR2 e TR3).
Até o sexto dia de armazenamento foi observado comportamento crescente no valor de $\mathrm{L}^{*}$, caracterizado pelo esbranquiçamento na superfície das rodelas, em seguida os valores decresceram até o final do experimento. $\mathrm{Na}$ Figura 1 (b) pode-se verificar que os maiores valores foram obtidos no TR3, o que indica que as rodelas de batata baroa submetidas a esse tratamento apresentavam coloração mais clara. Nunes et al. (2011), trabalhando com batatas baroa minimamente processadas, não verificaram efeito dos antioxidantes e tempo de armazenamento sobre o valor $\mathrm{L}^{*}$.

Pillon (2003), trabalhando com cenouras minimamente processadas sob refrigeração por 21 dias, verificou esbranquiçamento do produto desde o início até o final do período de armazenamento, o que alguns autores denominam de "white blush" e atestam ser resultado da desidratação das células superficiais, devido aos danos causados pelo processamento. Para outros autores, o esbranquiçamento se deve à formação de lignina na superfície do corte (BOLIN; HUXSOLL, 1991) ou então à combinação dos dois processos, a desidratação e formação de lignina (CINEROS-ZEVALLOS et al., 1995). Tal fato também pode ser atribuído ao ressecamento de resquícios celulares que permanecem na superfície do tecido. Como a batata baroa é rica em amido, e sendo este de coloração branca, após o corte este componente ficou aderido à superfície, conferindo aspecto esbranquiçado ao produto.

\section{REVENG}


Observou-se redução nos valores da coordenada $\mathrm{b}^{*}$ (Figura 2), sendo que, as rodelas submetidas ao TR3 foram as que tiveram maiores valores. Logo, essas rodelas foram as que apresentaram menor perda da coloração amarela, o que faz com que sejam mais aceitas pelos consumidores.

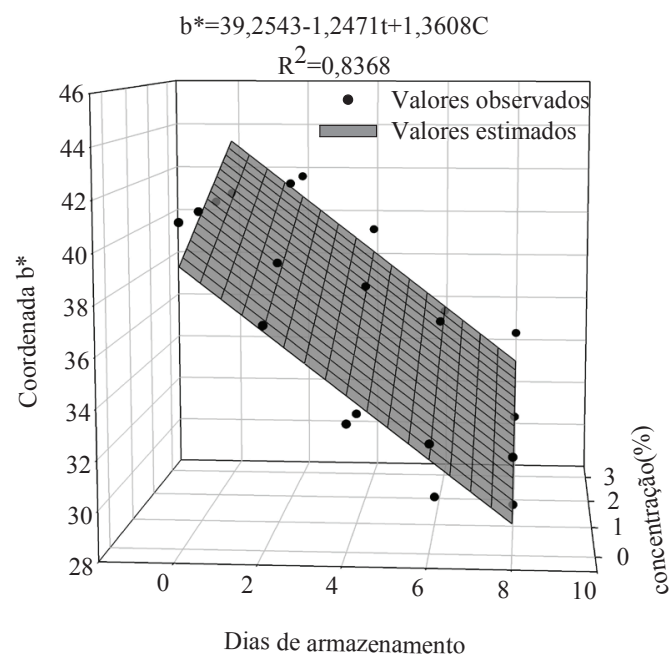

(a)

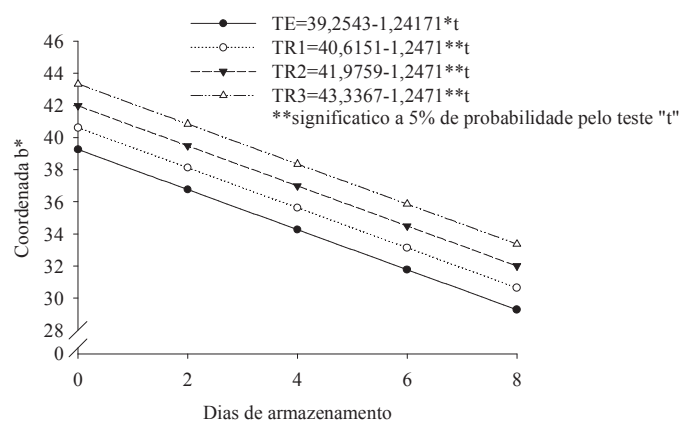

(b)

Figura 2. (a) Superfície de resposta da coordenada b*. (b) Cortes da superfície de resposta da coordenada $b^{*}$ para os tratamentos (TE, TR1, TR2 e TR3).

A perda da coloração amarela também pode ser explicada pelo Índice de Amarelecimento (YI). Este índice pode ser usado como parâmetro de qualidade do produto. Vasconcellos et al. (2011) verificaram que o YI foi uma alternativa viável para análise da qualidade de grãos de café armazenados.

Na Figura 3 é apresentado o comportamento do índice de amarelecimento ao longo do armazenamento.

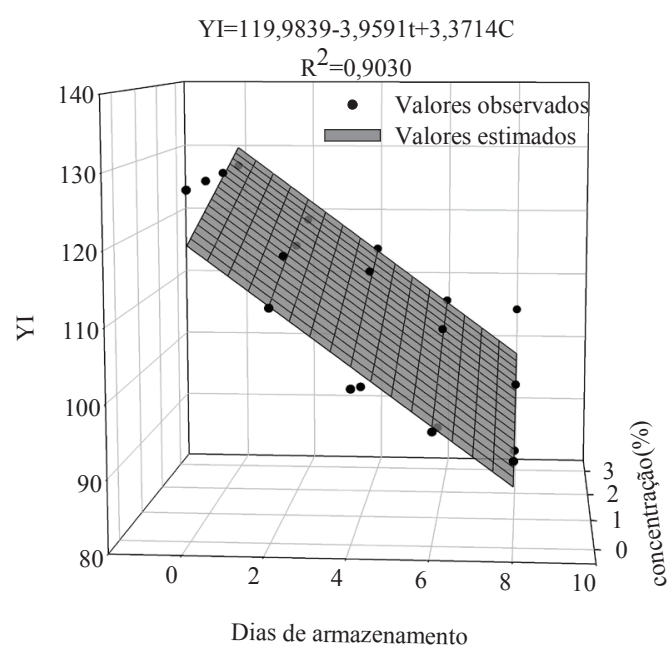

(a)

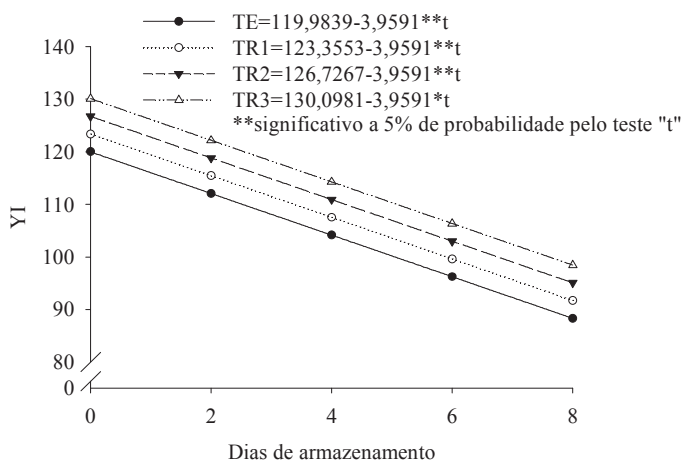

(b)

Figura 3. (a) Superfície de resposta do índice YI. (b) Cortes da superfície de resposta do índice YI para os tratamentos (TE, TR1, TR2 e TR3).

Assim como a coordenada $b^{*}$, o YI teve um comportamento decrescente ao longo do perído de armazenamento, indicando que as rodelas perderam parte da coloração amarela, afetando a qualidade final do produto. Com o tratamento TR3 foram mantidos os maiores valores, enquanto o tratamento testemunha proporcionou os menores.

Na Figura 4 é apresentado o comportamento dos índices colorimétricos croma (Cr*), ângulo hue $\left(\mathrm{h}^{*}\right)$ e diferença total de cor $\left(\Delta \mathrm{E}^{*}\right)$ ao longo do perído de armazenamento.

O decréscimo no índice colorimétrico croma ao longo do período de armazenamento é um indicador da diminuição de tonalidade ou matiz da coloração, em que menores valores levam a cores menos puras, devido, provavelmente, ao aparecimento de outros pigmentos. Dessa forma, o tratamento TR3, por ter proporcionado menor decréscimo desse índice, mostrou melhor comportamento, gerando rodelas com coloração mais homogêneas. 

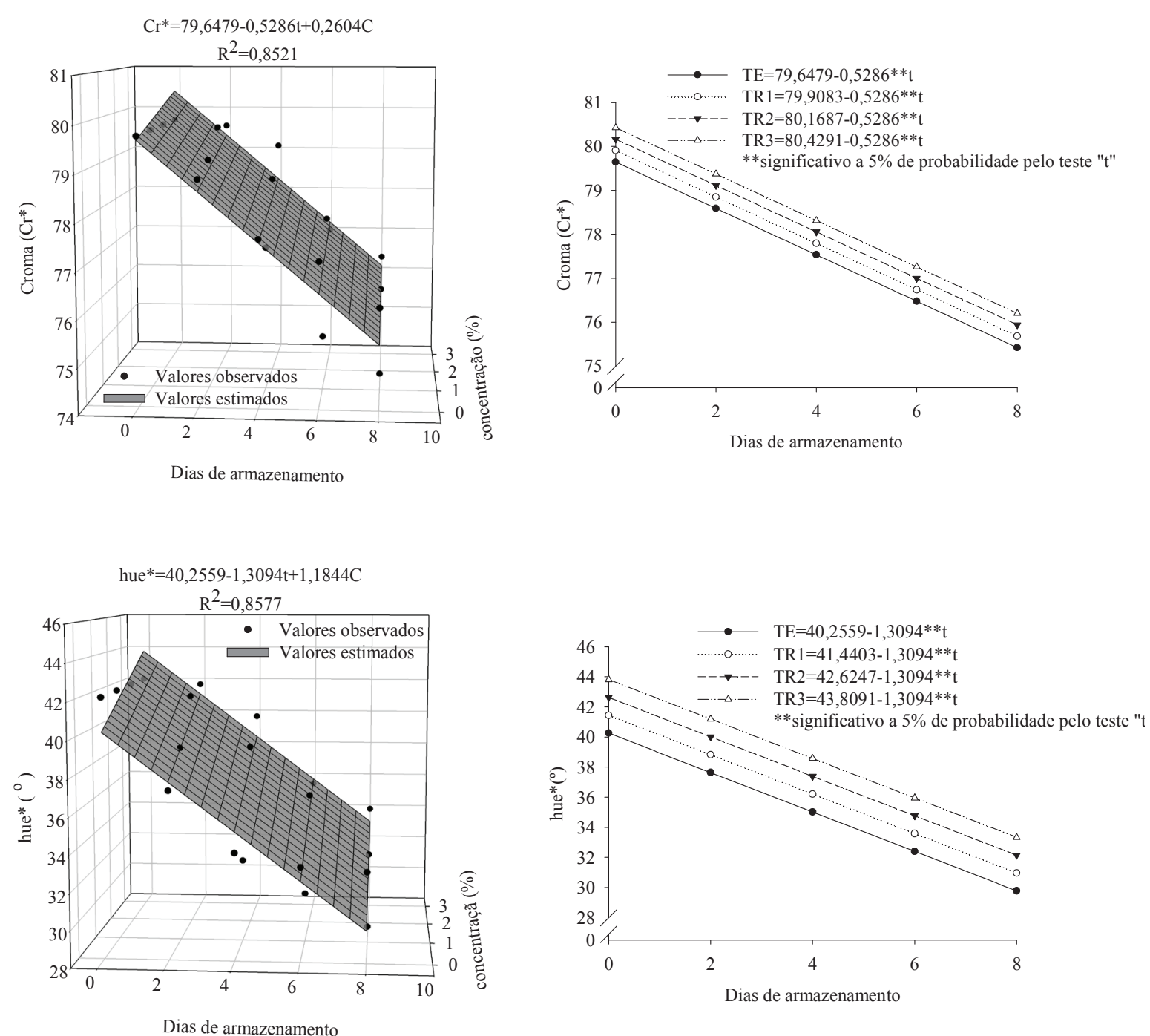

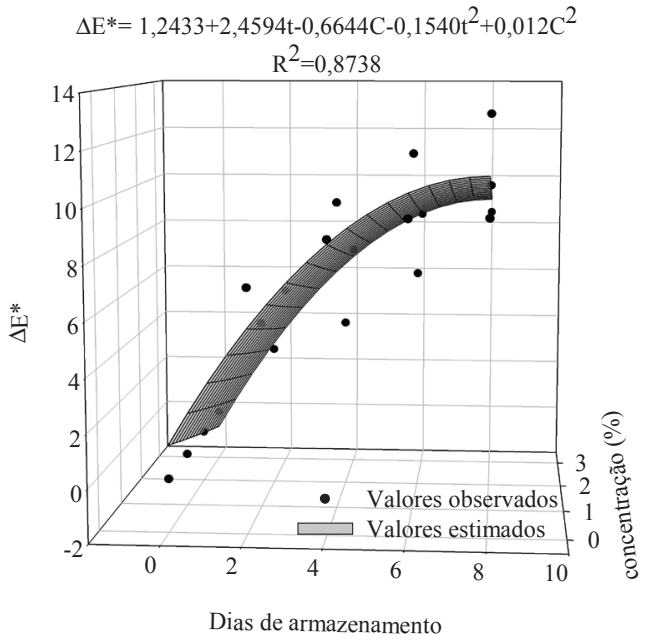

(a)

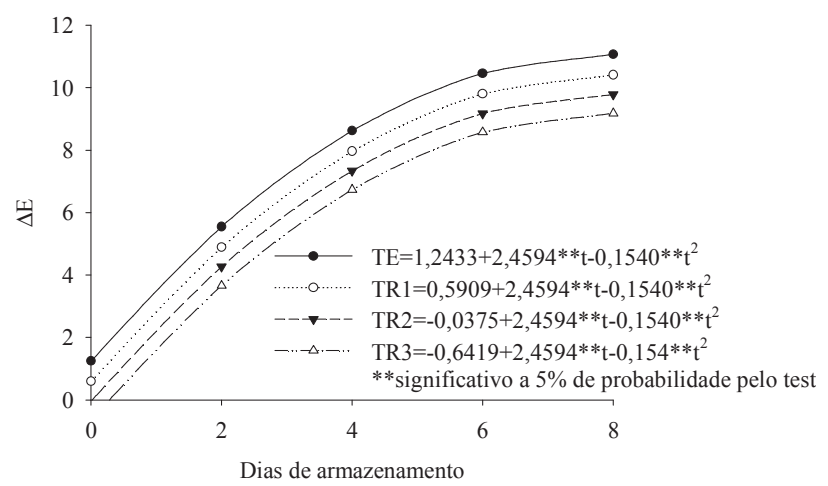

(b)

Figura 4. (a) Superfícies de resposta dos índices $C^{*}$, $h *$ e $\Delta E$. (b) Cortes das superfícies de resposta dos índices $\mathrm{Cr}^{*}, \mathrm{~h}^{*}$ e $\Delta \mathrm{E}$ para os tratamentos (TE, TR1, TR2 e TR3). 
O comportamento decrescente do índice ângulo hue ao longo do armazenamento sugere uma mudança na coloração das rodelas do amarelo para o azul. Essa alteração foi devida ao surgimento das pontuações escuras. O TR 3 foi o tratamento que, ao final dos 8 dias de armazenamento, manteve o maior valor do $h^{*}$.

Em relação ao índice diferença total de cor $(\Delta \mathrm{E})$, as coordenadas $\mathrm{L}^{*} \mathrm{e} \mathrm{a}^{*}$ tiveram uma variação de $10,1 \%$ e $10,8 \%$, respectivamente. Já na coordenada $b^{*}$, a variação chegou a $29,4 \%$ quando empregado TR1 no oitavo dia de armazenamento (Quadro 1). Portanto, visto que a coordenada $b^{*}$ foi a que mais influenciou nos valores de $\Delta \mathrm{E}$, este índice representou a variação da coloração amarela nas rodelas de batatas baroa ao longo do período de armazenamento. $\mathrm{O}$ tratamento TR3 proporcionou, em média, os menores valores, indicando perda da coloração amarela menos intensa e mais lenta.

No que se refere ao índice de escurecimento (IE), foi observado uma diminuição dos valores ao longo do armazenamento em todos os tratamentos aplicados (Figura 5).

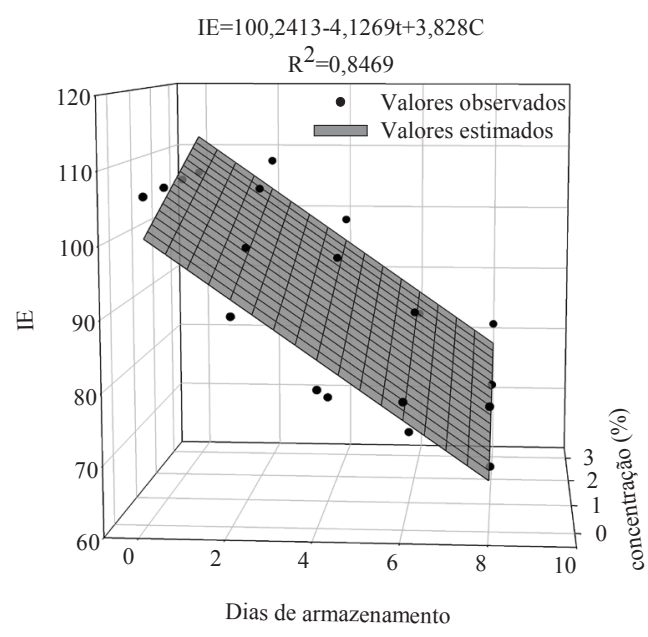

(a)

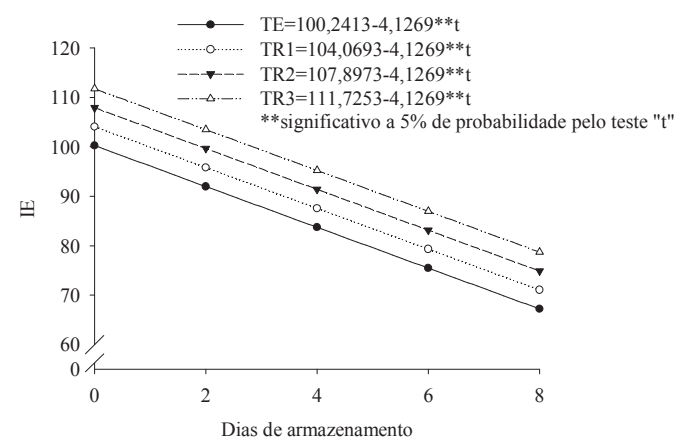

(b)

Figura 5. (a) Superfície de resposta do IE. (b) Cortes das superfícies de resposta do IE para os tratamentos (TE, TR1, TR2 e TR3).
Esses resultados são semelhantes aos encontrados por Medeiros (2009) que, em estudos sobre deterioração pós-colheita da mandioca minimamente processada também verificou decréscimo dos valores de IE. Porém, diferem dos encontrados por Pineli et al. (2005) que observaram aumento no IE ao longo do período de armazenamento de batatas 'Ágata' minimamente processadas e embaladas em náilon multicamadas.

Os resultados da variação do $\mathrm{pH}$ nas rodelas de batatas baroa durante o perído de armazenamento estão apresentados na Figura 6.

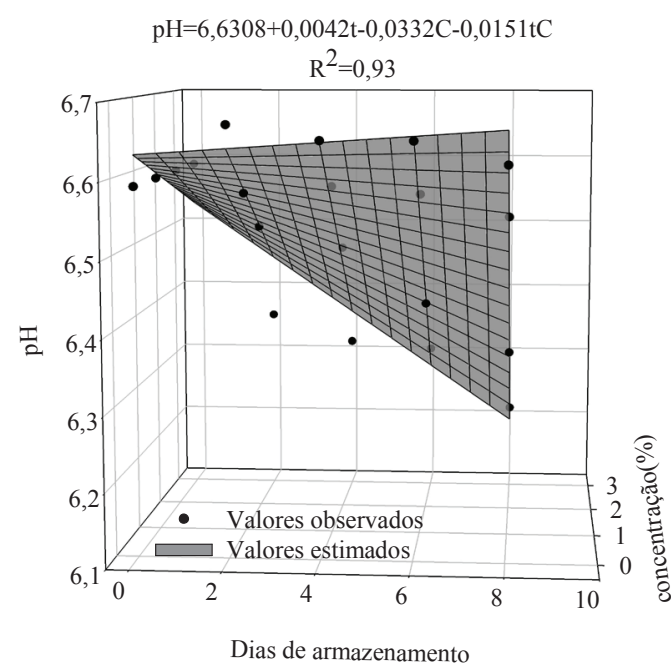

(a)

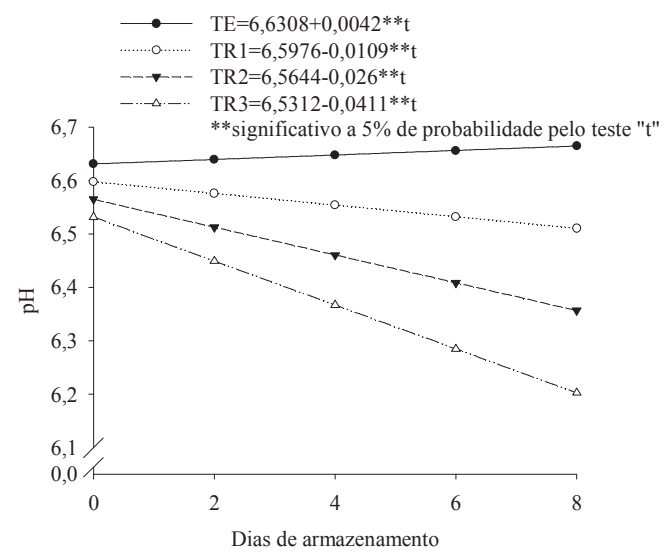

(b)

Figura 6. (a) Superfície de resposta do pH. (b) Cortes da superfície de resposta do $\mathrm{pH}$ para os tratamentos (TE, TR1, TR2 e TR3).

$\mathrm{O} \mathrm{pH}$ apresentou valor inicial de 6,59, chegando ao mínimo de 6,22. Essa variação foi pequena e o pH se manteve dentro da faixa recomendada de 
5,5 a 7 para frutas e hortaliças frescas (VANETTI, 2000). Os resultados obtidos são semelhantes aos encontrados por Oliveira et al. (2003), que atribuíram a redução no $\mathrm{pH}$ em mandioca minimamente processada ao uso de ácido cítrico como antioxidante.

Os valores de sólidos solúveis totais (SST) tiveram aumento nos primeiros dias de armazenamento, porém a partir do quarto dia foi observado comportamento decrescente até o final do período experimental. $\mathrm{Na}$ Figura 7 verifica-se a evolução destes valores ao longo do período de armazenamento.

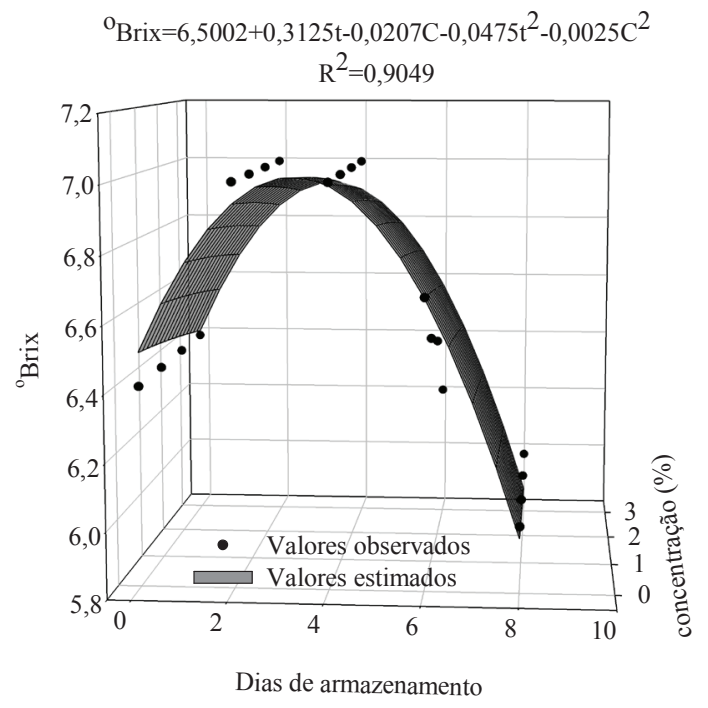

(a)

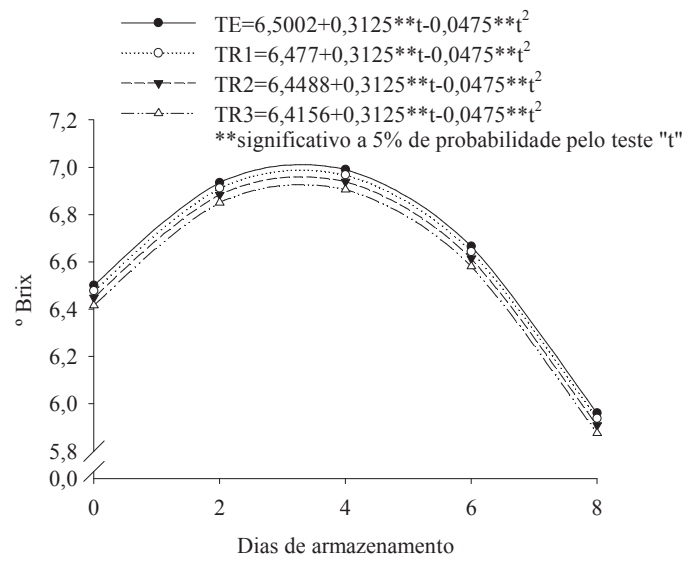

(b)

Figura 7. (a) Superfície de resposta de SST. (b) Cortes da superfície de resposta de SST para os tratamentos (TE, TR1, TR2 e TR3).

Normalmente os sólidos solúveis aumentam ao longo do período de armazenamento devido à transformação do amido em açúcar. Porém, neste experimento, foi observado o contrário. Uma possível explicação para este comportamento está relacionada ao estresse mecânico provocado pelo processamento mínimo. $\mathrm{O}$ açúcar é consumido nos processos respiratório e fermentativo, com produção de $\mathrm{CO}_{2}$, água e ácidos orgânicos, respectivamente. Tais processos contribuem para a redução dos sólidos solúveis com o tempo, cujos valores estão associados à diferença entre liberação e degradação de açúcares (PINELI et al., 2005).

O tempo de armazenamento teve um efeito significativo na perda de água das rodelas de batatas baroa. Conforme pode ser observado na figura 8 .

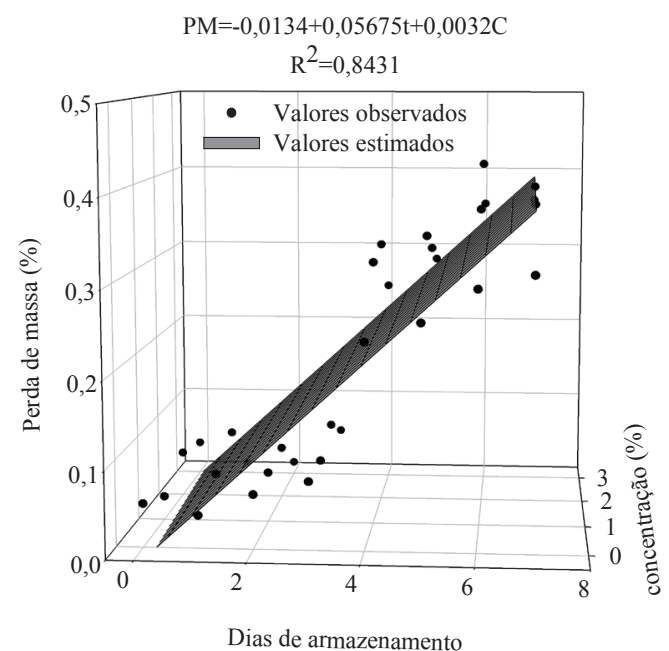

(a)

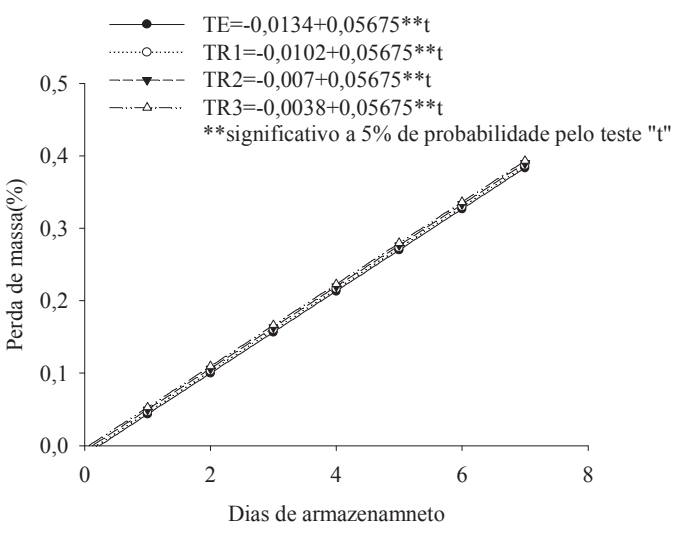

(b)

Figura 8. (a) Superfície de resposta da perda de massa. (b) Cortes da superfície de resposta da PM para os tratamentos (TE, TR1, TR2 e TR3). 
A perda de massa das rodelas foi pequena durante todo o período de conservação, não sendo possível distinguir as curvas de cortes referentes a cada tratamento. Segundo Chitarra e Chitarra (2007) perdas de até $3 \%$ são aceitáveis, valores acima disso deixam o produto com aspecto murcho ou ressecado, interferindo na qualidade visual e, consequentemente, sua rejeição pelo consumidor. Portanto, não foi verificada influência clara da aplicação de ácido nas batatas baroa minimamente processada na perda de massa ao longo do tempo de seu armazenamento.

\section{CONCLUSÕES}

- A batata baroa cultivar Amarela de Carandaí minimamente processada apresentou melhor qualidade quando submetida ao tratamento com 3\% ácido ascórbico $+3 \%$ ácido cítrico, caracterizando um produto com maiores valores de $b^{*}$ e YI, permitindo que as rodelas chegassem ao final do período de armazenamento com menor perda da coloração amarela

\section{REFERÊNCIAS BIBLIOGRÁFICAS}

ANDRADY, A.L.; TORIKAI, A. Photoyellowing of mechanical pulps III. Intensity effects and doseresponse relationships. Polymer Degradation and Stability, v.66, p.317-322, 1999.

ARRUDA, M.C.; JACOMINO, A.P.; FILLET, M.H.; GALLO, C.R.;MORETTI, C.L. Conservação de melão rendilhado minimamente processado sob atmosfera modificada ativa. Ciência e Tecnologia de Alimentos, v.24, n.1, p.53-58, 2004.

ASSOCIATION OF OFFICIAL ANALYTICAL CHEMISTS. Official methods of analysis of AOAC international. 16ed. AOAC International, 1997.

BOLIN, H.R.; HUXSOLL, C.C. Control of minimally processed carrot (Daucus carota) surface discoloration caused by abrasion peeling. Journal of Food Science, v.56, n.2, p.416-418, 1991.

CHITARRA, M.I.F.; CHITARRA, A.B. Processamento mínimo de alface. In: Manual de
Processamento Mínimo de Frutas e Hortaliças. Brasília: Embrapa Hortaliças e SEBRAE. 531p. 2007.

CINEROS-ZEVALLOS, L.; SALVEIT, M.E.; KROCHTA, J.M. Mechanism of surface white discoloration of peeled (minimally processed) carrots during storage. Journal of Food Science, v.60, n.2, p.320-324, 1995.

HAMINIUK， C.W.I.; OLIVEIRA， C.R.G.; BAGGIO, E.C.R.; MASSON, M.L. Efeito de prétratamentos no escurecimento das cultivares de maçã Fuji e Gala após o congelamento. Ciência e Agrotecnologia, v.29, p.1029-1033, 2005.

MEDEIROS, E.A.A. Deterioração pós-colheita da mandioca minimamente processada. 2009. 101f. Tese (Doutorado em Fisiologia Vegetal) Departamento de Biologia Vegetal, Universidade Federal de Viçosa, Viçosa (MG), 2009.

MORETTI, C.L. Manual de processamento mínimo de frutas e hortaliças. Brasília: Embrapa Hortaliças, 2007.

NUNES, E.E.; VILAS BOAS, E.V.B.; XISTO, A.L.R.P. Qualidade de mandioquinha-salsa minimamente processada: Uso de antioxidantes. Journal of Biotechonoly and Biodiversity, v.2, n.3, p.43-50, 2011.

OLIVEIRA, M.A.; PANTAROTO, S.; CEREDA, M.P. Efeito da sanitização e de agente antioxidante em raízes de mandioca minimamente processadas. Brazilian Journal of Food Technology, v.6, p.339-344, 2003.

PALOU, E.; LÓPEZ-MALO, A.; BARBOSACÁNOVAS, G.V.; WELTI-CHANES, J.; SWANSON, B.G. Polyphenoloxidase activity and color of blanched and high hydrostatic pressure treated banana puree. Journal of Food Science, v.64, p.42-45, 1999.

PILLON, L. Estabelecimento da vida útil de hortaliças minimamente processadas sob atmosfera modificada e refrigeração. 2003. 111f. Dissertação (Mestrado em Ciências) ESALQ, Piracicaba, SP. 
PINELI, L.L.O.; MORETTI, C.L.; ALMEIDA, G.C.; ONUKI, A.C.A.; NASCIMENTO, A.B.G. Caracterização química e física de batatas "Ágata"minimamente processadas, embaladas sob diferentes atmosferas ativas. Pesquisa Agropecuária Brasileira, v.40, p.1035-1041, 2005.

SCALON, S.P.Q.; ZÁRATE, N.A.H.; VIEIRA, M.C. Embalagem e temperatura na manutenção da qualidade pós-colheita de mandioquinha-salsa. Ciência e Agrotecnologia, v.26, p.559-564, 2002.
VANETTI, M.C.D. Controle microbiológico e higiene no processamento mínimo. In: Encontro Nacional de Processamento Mínimo de Frutas e Hortaliças, Anais... UFV: Viçosa, MG, 2000.

VASCONCELLOS, D.S.L.; CORREAA, P.C.; AFONSO JÚNIOR, P.C.; BAPTESTINI, F.M.; CAMPOS, S.C. Utilização do índice de amarelecimento como fator qualitativo em grãos de café armazenados. VII Simpósio de Pesquisa dos Cafés do Brasil. Anais... Araxá, 2011. 